\title{
Prevalence of diffuse idiopathic skeletal hyperostosis (DISH) assessed with whole- spine computed tomography in 1479 subjects
}

\author{
Akihiko Hiyama*, Hiroyuki Katoh, Daisuke Sakai, Masato Sato, Masahiro Tanaka and Masahiko Watanabe
}

\begin{abstract}
Background: Computed tomography (CT) analyses have reported that the prevalence of diffuse idiopathic skeletal hyperostosis (DISH) in Japan is 8.7-27.1\%. However, these data were obtained using chest-abdominal $\mathrm{CT}$, and no evaluations of sagittal, coronal, and axial images using whole-spine $C T$ have been reported. The aim of this study was to investigate the prevalence and characteristic of DISH by whole spinal CT.

Methods: Participants were patients who had experienced trauma who had undergone whole-spine CT scanning based on the initial clinical practice guidelines for trauma in our institute from April 2015 to February 2018. The subjects were $>20$ years old and 1479 were included in the analysis. The presence and distribution of DISH and clinical parameters such as age and sex were reviewed retrospectively according to the location of DISH.

Results: The overall prevalence of DISH was $19.5 \%(n=289)$. Subjects with DISH were older than those without. DISH was located in the thoracic spine in $65.1 \%$ and thoracolumbar spine in $24.2 \%$ of patients. More than $80 \%$ of ligamentous ossifications associated with DISH occurred at T8 $(n=255,88 \%)$, T9 $(n=262,91 \%)$, and T10 ( $n=247$, $85 \%)$. Most of the ossification occurred to the right anterior of the vertebral body, and there were few ossifications in the areas in contact with the artery and vein.

Conclusions: The prevalence of DISH based on whole-spine CT was 19.5\%. Ossification was noted more often at T8, T9, and T10, and to the right anterior of the vertebral body. It is for the first time report that we have studied the location of ossification in detail using the axial images of whole spine CT. We hope this study will enhance the understanding of the characteristics of DISH.
\end{abstract}

Keywords: Diffuse idiopathic skeletal hyperostosis (DISH), Computed tomography (CT), Prevalence, Thoracic spine

\section{Background}

Recently, much attention has focused on vertebral fractures accompanying diffuse idiopathic skeletal hyperostosis (DISH). That is, an ankylosed spine tends to fracture as result of minor injuries such as falling because of stress concentration. Such fractures can cause reverse chance fractures that can lead to spinal paralyses, and are often resistant to conservative treatment [1-3]. Like other conditions with ligament ossification, DISH is thought to result from a multifactorial process whose

\footnotetext{
* Correspondence: a.hiyama@tokai-u.jp

Department of Orthopaedic Surgery, Tokai University School of Medicine, 143 Shimokasuya, Isehara, Kanagawa 259-1193, Japan
}

etiology is not easily identified. In Japan, which is experiencing an aging society, the prevalence of minor paralysis caused by trauma is on the rise.

Proposed by Resnick et al. in 1975, DISH is a degenerative disease in which the spinal longitudinal ligaments and entheses gradually become ossified [4]. The pathogenetic mechanisms responsible for DISH are poorly understood, but genetic, metabolic, endocrine, anatomic, environmental, and toxic factors may contribute to its' development $[5,6]$. Moreover, DISH is a condition of the elderly and is rarely seen before middle age. It is more common in males than in females with male/female ratios ranging between $2: 1$ and $7: 1$ [7, 8]. The 
criteria most often used to diagnose DISH was first reported by Resnick and Niwayama in 1976 [9], before computed tomography (CT) was available as a diagnostic tool. However, when examining the prevalence of $\mathrm{DISH}$, the problem is that the criteria also differs according to the article $[9,10]$.

The prevalence of DISH reported both in Japan and internationally varies widely between $3.8-25 \%$ [8, 9, 11-15]. It is considered to occur more frequently in Caucasians than in people of Asian and Black ethnicity. However, some groups have suggested that this considerable variation could be attributed to the incomplete diagnosis of DISH based on X-ray alone. CT provides far more detailed imaging of the intervertebral disc spaces and bridging ossifications, but there are only a few reports on CT-based diagnosis of DISH. Analyses using CT have reported that the prevalence of DISH in Japan averages $8.7 \%$ (13\% for males, $2.5 \%$ for females) and may be as high as $27.1 \%$ (38.7\% for males, $13.9 \%$ for females) [12, 16]. However, these data were obtained using chest-abdominal CT, and evaluations using sagittal, coronal, and axial whole-spine CT have not been reported. Most of the patients surveyed so far have been older than 40 years and, therefore, the true prevalence is unknown. The purpose of this study was to use whole-spine CT to investigate the prevalence and characteristics of DISH in trauma patients examined in an emergency critical care setting. In addition, we hope this study will enhance the understanding of the characteristics of DISH.

\section{Methods}

Our institution is a tertiary emergency medical facility that treats over 800 patients with severe trauma a year. The patients included in this study were those who had undergone whole-spine CT scanning during the period between April 2015 to February 2018 based on the clinical practice guidelines for trauma patients at our institute. The patients were aged over 20 years and their mean age was 54.7 years.

A total of 1479 subjects were included in the analysis: 1023 males and 456 females. The presence and distribution of DISH, and their clinical parameters such as age and sex were reviewed retrospectively and classified according to the location of DISH. The numbers of subjects in the age groups $20-39,40-49,50-59,60-69$, $70-79,80-89$, and $\geq 90$ years were described in Table 1 .

\section{Computed tomography (CT) examination}

All CT scans were performed on a multidetector CT (120 kV, $380 \mathrm{~mA}, 0.6 \mathrm{~mm}$ slice; SOMATOM Definition AS; Siemens Healthcare, Forchheim, Germany) equipped with a 128-slice multidetector array. The diagnosis of DISH was based on whole-spine CT according to the criteria proposed by Resnick and Niwayama [9]: flowing
Table 1 Distribution of study population according to sex

\begin{tabular}{llll}
\hline Age group, yrs & Male & Female & ALL \\
\hline $20-39$ & 286 & 93 & 379 \\
$40-49$ & 167 & 60 & 227 \\
$50-59$ & 160 & 46 & 206 \\
$60-69$ & 167 & 70 & 237 \\
$70-79$ & 162 & 98 & 260 \\
$80-89$ & 70 & 77 & 147 \\
$90-$ & 11 & 12 & 23 \\
& 1023 & 456 & 1479 \\
\hline
\end{tabular}

ligamentous ossification in $\geq 4$ contiguous vertebrae of the spine, with preserved intervertebral disc space, and the absence of inflammatory changes in the apophyseal joints or the sacroiliac region. The reconstructed CT sagittal, coronal, and axial images (Fig. 1) were used rather than X-ray to evaluate the continuous ligament ossification. All CT data were evaluated by authors. For testing the reliability of diagnoses, the testers read the same images of 20 patients to check inter-observer agreement. We also used intra-observer error at different time points, and the interval was found to be longer than one month by the first author. Differences were settled by consensus to minimize intra- and inter-observer bias and errors. Finally all images were reviewed by the first author.

\section{Statistical analysis}

Statistical analysis was performed using SPSS software for Windows (v. 20.0; IBM Corp., Armonk, NY, USA). All values are expressed as mean \pm standard deviation. We studied statistical differences between the group with and without DISH. Analysis of DISH prevalence in males and females was performed using the chi-squared test. Analysis of variance with a post hoc test (MannWhitney $U$ test) was used for comparisons. P-values $<0.05$ were considered significant.

\section{Results}

The Kappa coefficient of inter- and intra-observer agreements were 0.79 and 0.89 , respectively.

The demographics of the subjects included in this study are shown in Table 2. The prevalence of DISH was $19.5 \%(289 / 1479)$. The subjects with DISH were significantly older than those without: 71.7 and 50.6 years, respectively $(P<0.001)$. The prevalence of DISH increased with age. The prevalence of DISH in subjects aged $\geq 70$ years was $40.9 \%$ (176/430), indicating that one in two people $\geq 70$ years had DISH. In addition, the prevalence of DISH increased with age in both males and females (Fig. 2a). The results showed that the prevalence rates classified by age groups 20-39, 40-49, 50-59, 60-69, 

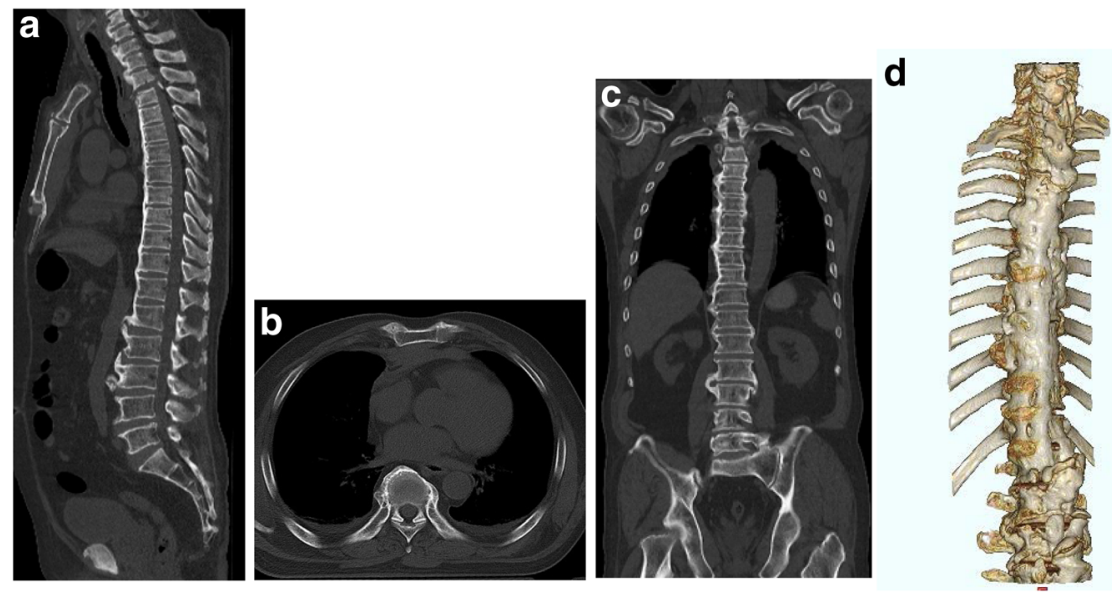

Fig. 1 CT images of a patient with DISH. Reconstructed CT of sagittal (a), axial (b), coronal (c) and 3D (d) views in a patient with DISH

$70-79,80-89$, and $\geq 90$ years were $0.3,4.8,18.1,32.3$, $50.0,51.4$, and $63.6 \%$ in male and $0,3.3,10.9,20.0,19.4$, 36.4 , and $41.7 \%$ in female, respectively. There were a significantly greater number of subjects with DISH in their 70 s using the chi-squared test.

Among the 289 subjects with DISH, 216 (prevalence $216 / 1023=21.1 \%)$ were male and $73(73 / 456=16.0 \%)$ were female (Fig. 2b). This tendency for a higher frequency in male was a significant $(P<0.05)$. In addition, when analyzed with subjects $\geq 40$ years as in previous reports, the prevalence of DISH in 737 males and 363 females was $29.2 \%(215 / 737)$ and $20.1 \%$ (73/363), respectively, also showing a significant sex difference $(P<0.01)$ (data not shown).

Table 2 Distribution of study population according to DISH(+) or (-)

\begin{tabular}{llll}
\hline & DISH (+) & DISH (-) & ALL \\
\hline Sex & $\mathrm{N}$ & $\mathrm{N}$ & $\mathrm{N}(\%)$ \\
Male & 216 & 807 & $1023(69.2)$ \\
Female & 73 & 383 & $456(30.8)$ \\
Male to Female & $3.0: 1$ & $2.1: 1$ & $2.3: 1$ \\
Age, Mean & 71.7 & 50.6 & 54.7 \\
Age group, yrs & $\mathrm{N}(\%)$ & $\mathrm{N}(\%)$ & $\mathrm{N}$ \\
20-39 & $1(0.3)$ & $378(99.7)$ & 379 \\
$40-49$ & $10(4.4)$ & $217(95.6)$ & 227 \\
$50-59$ & $34(16.5)$ & $172(83.5)$ & 206 \\
$60-69$ & $68(28.7)$ & $169(71.3)$ & 237 \\
$70-79$ & $100(38.5)$ & $160(61.5)$ & 260 \\
$80-89$ & $64(43.5)$ & $83(56.5)$ & 147 \\
$90-$ & $12(52.2)$ & $11(47.8)$ & 23 \\
Total (\%) & $289(19.5)$ & $1190(80.5)$ & 1479 \\
\hline
\end{tabular}

DISH diffuse idiopathic skeletal hyperostosis, $N$ number
We classified DISH into six types according to location in which ossification along the aspect of $\geq 4$ contiguous vertebral bodies was observed: (1) cervicalossification only in the cervical region (C1-C7); (2) cervicothoracic-ossification only in the cervicothoracic region (C1-T12); (3) thoracic-ossification only in the thoracic region (T1-T12); (4) thoracolumbar-ossification only in the thoracolumbar region (T1-L5); (5) lumbar-ossification only in the lumbar region (L1-L5); and (6) whole spine-ossification in the entire spine (C1-L5). Figure 3 shows the prevalence of DISH classified by location in the spine: $65.1 \%$ were thoracic, and $24.2 \%$ were thoracolumbar. The prevalence of cervical and lumbar spine involvement was low.

Evaluation of the fused segments from the cervical to lumbar spine for all subjects revealed that the eighth thoracic (T8) to the 10th thoracic (T10) levels were the levels most involved, with more than $80 \%$ of the ligamentous ossifications associated with DISH located at T8 ( $n=255$ segments/289subjects, $88 \%)$, T9 ( $n=262$ segments/289subjects, 91\%), or T10 $(n=247$ segments/ 289subjects, 85\%) (Fig. 3). Of the 233 cases with consecutive ossification at T8-T10, the apex of thoracic kyphosis was located at T8-T10 in 142 subjects (60.9\%) (Table 3).

Axial images of the ossification lesions at T9 (Fig. 4a) were studied further focusing on the location of the ossification, revealing that the ossification at T9 was observed in almost all cases in area 1, which is the right anterior region of the vertebra (98.3\%) (Fig. 4b). As age increased, ossification in the left anterior region also appeared (Table 4). In a few subjects, the ossification was observed in the area in contact with the aorta and the azygous vein (Fig. 4c). The average number of vertebral bodies continuously bridged by ossification of the anterior longitudinal ligament was 7.9, and involvement of 


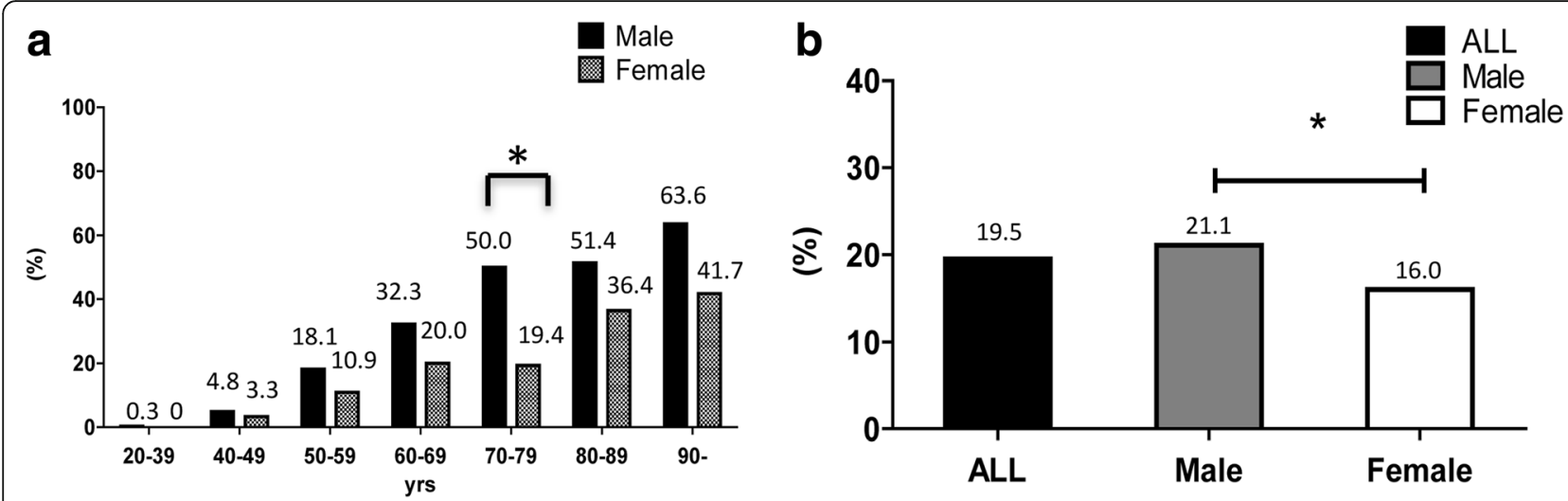

Fig. 2 Prevalence of DISH detected by CT. a Age distribution of the prevalence of DISH detected by CT. The prevalence of DISH increased with age in both males and females. $\mathbf{b}$ Comparison of the prevalence of DISH between male and female

$\geq 12$ vertebrae was seen in 47 of the 289 patients (16.3\%). The average numbers of ossified vertebrae according to the age groups 20-49 $(n=11), 50-59 \quad(n=34)$, 60-69 (n=68), 70-79 $(n=100), 80-89 \quad(n=64)$, and $\geq 90 \quad(n=12)$ years were $6.8 \pm 2.0,6.8 \pm 2.6,7.4 \pm 3.2$, $8.3 \pm 3.5,8.3 \pm 4.2$, and $9.4 \pm 3.6$, respectively, showing that the number of vertebrae with ligamentous ossification increased with age.

\section{Discussion}

DISH is a common condition in the elderly, but DISH is still insufficiently investigated and understood. In this study, CT images of the whole spine were used to determine the prevalence of DISH in 1479 patients aged $>20$ years. While the prevalence of DISH has been reported to vary widely from 3.8 to $25 \%$, it was $19.5 \%$ in these subjects. Factors that may affect the prevalence of DISH include differences in ethnic and genetic background, diagnostic imaging methods, patient age, and lifestyle habits. The prevalence of DISH in Japan, which would exclude the effects of race and genetic factors, has been reported to range from 8.7 to $27.1 \%$ [12, 13, 16]. Based on chest X-rays of 1363 patients, Weinfeld et al. showed that the prevalence differed between ethnic groups [14]. They reported DISH to be less common in the black, Native-American and Asian populations.

The prevalence of DISH may differ between past and present reports because of advances in diagnostic imaging (Additional file 1). In past reports, DISH was identified by X-ray alone, which resulted in lower prevalence than that identified by recent reports of DISH identified through examination of chest CT. Hirasawa et al. compared the prevalence of DISH evaluated by reconstructed $\mathrm{CT}$ of the chest to pelvis against plain radiography of the chest and abdomen [12], and found that the prevalence of DISH based on CT was $27.1 \%$ and that based on X-ray was $17.6 \%$. Mori et al. reported that the prevalence of DISH was $8.7 \%$ using chest CT performed for investigation of pulmonary disease [16], which was smaller than that reported by Hirasawa et al. [12]. The

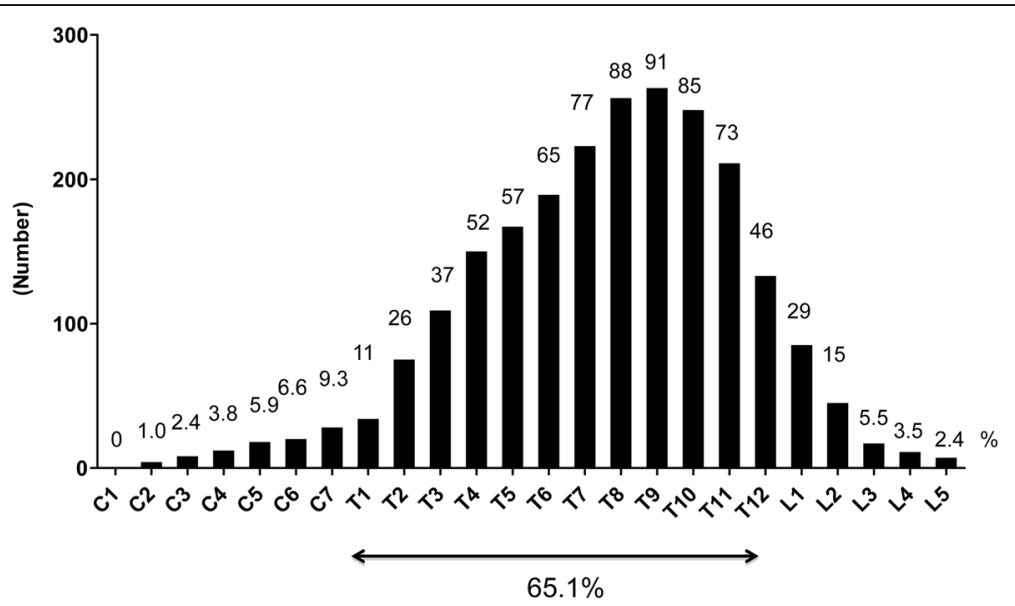

Fig. 3 Prevalence of DISH classified by location in the spine. The levels of fused segments are shown from the cervical to lumbar spine, and involved mainly T8-10 
Table 3 Among 233 subjects who had consecutive ossification in T8 - T10, 142 subjects were found at the apex of the thoracic kyphosis at T8 - T10

\begin{tabular}{llll}
\hline Apex of kyphosis & $N$ & $\%$ & \\
\hline T5 & 1 & 0.4 & \\
T6 & 11 & 4.7 & \\
T7 & 37 & 15.9 & $142(60.9 \%)$ \\
T8 & 68 & 29.2 & \\
T9 & 47 & 20.2 & \\
T10 & 27 & 11.6 & \\
T11 & 25 & 10.7 & \\
T12 & 11 & 4.7 & \\
L1 & 5 & 2.1 & \\
L3 & 1 & 0.4 \\
\end{tabular}

$N$ number

differences in prevalence between these studies may relate to the sample size: that Mori et al. evaluated a large number of subjects (3013 cases), but was a patient-based and not a population-based study [16]. They acknowledged that the relationship between pulmonary disease and ossification of the spinal ligaments, including DISH, could have affected the prevalence data. Moreover, the study by Mori et al. included only chest $\mathrm{CT}$ and was not based on $\mathrm{CT}$ of the chest to pelvis, which may account for the differences from the study by Hirasawa et al. [12]. Our findings and those of previous reports indicate that most cases of DISH occur in the middle and lower thoracic spine. This means that when making the diagnosis, evaluation using whole-spine CT will be more effective than X-ray $[12,16]$. On the accuracy of DISH diagnosis by $\mathrm{CT}$, intra- and inter-observer error by review of CT was also less than that of X-ray [12]. From these data, we think that CT may be a most suitable modality for the evaluation of DISH. Thus, analysis of the prevalence of DISH and its characteristics from the analysis using CT is considered to be useful for analyzing the treatment and pathology.

Another possible reason for the difference in prevalence of DISH is age of the study population. Most previous reports only included people older than 40 years with an average of 65 years $[8,13,17]$, whereas in this study, we included younger patients ( $>20$ years) for an average age of 54.7 years old.

One limitation of our study is that the patients' past history was not analyzed; in particular, the presence of metabolic disease, because the distribution of these patients may affect the prevalence of DISH. Although the pathogenesis of DISH has not been fully elucidated, DISH may be related to modern lifestyle-related diseases such as obesity and metabolic syndrome, and the prevalence rate of DISH may be higher in those with metabolic disease. Some have suggested that its prevalence

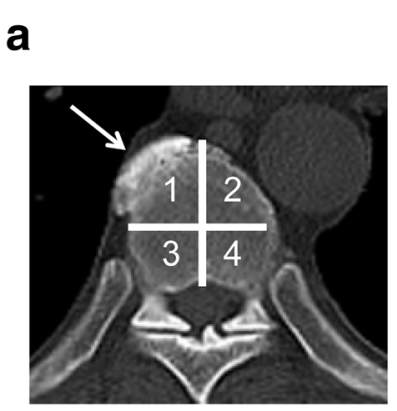

C

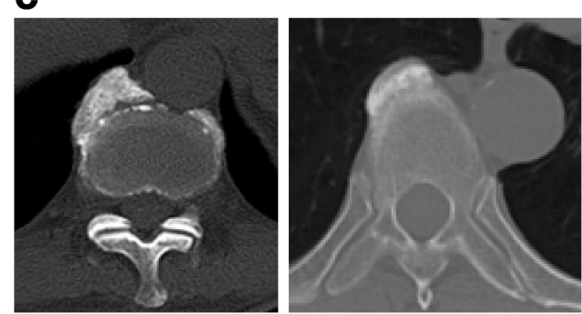

b
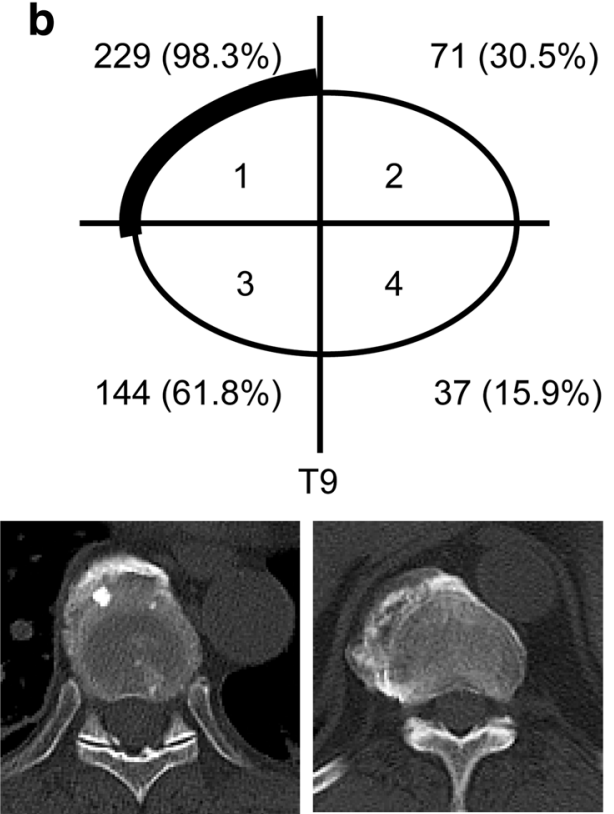

Fig. 4 Axial $(A X)$ image of the areas showing ossification at T9. a A 58-year-old man presented with DISH between T7-11. The vertebral body was divided into 4 areas from the AX image of T9. In this case ossification was observed in area 1 (arrow). b Location of ossification on vertebral body: Area 1, right anterior; Area 2, left anterior; Area 3, right posterior; Area 4, left posterior. c Ossification was rarely found in vertebral bodies contacting with the aorta and the azygous vein 
Table 4 Axial image of the lesion of ossification at the T9 level among 233 subjects who had consecutive ossification in T8 - T10

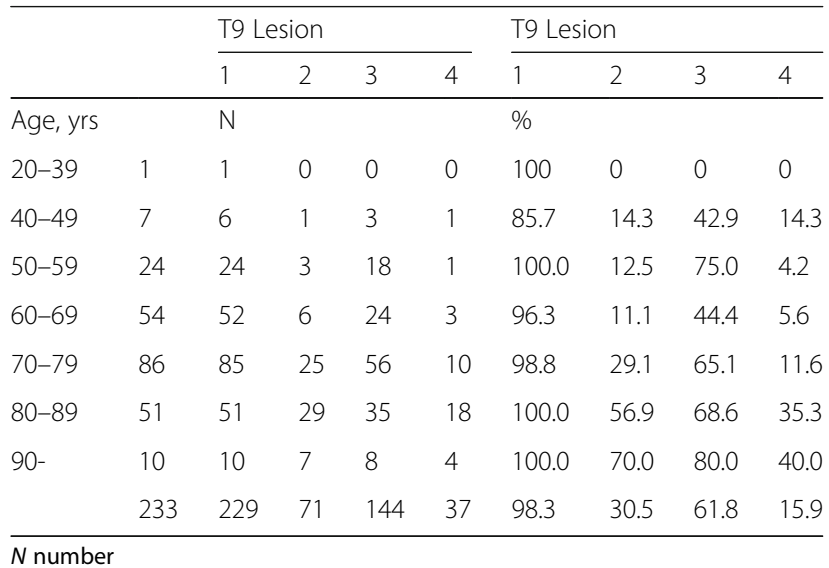

may increase in the coming decades because of the relationship between DISH and such modern lifestyle-related diseases $[5,6,18,19]$. We could not investigate any possible associations between such factors and DISH because no clinical background information except for age and sex were available in our retrospective study.

Other studies have reported a higher prevalence of DISH in males than in females. The Research on Osteoarthritis/Osteoporosis Against Disability (ROAD) study of Kagotani et al. investigated the prevalence of DISH in 1647 people who underwent whole-spine X-ray [13]. Their logistic regression analysis revealed that the prevalence of DISH was associated with male sex (odds ratio (OR): 5.55) and presence of severe lumbar spondylosis (OR: 5.50). It appears that male tend to be more likely to develop DISH than female regardless of ethnicity or genetic factors. In our study, there was a tendency for the prevalence of DISH to be higher in males than in females, but the difference was small than in previous studies. It is possible that this result may have been affected by the inclusion of younger patients than in previous studies.

The most common site of DISH is the thoracic spine. We found that most of the ossification occurred in the middle and lower thoracic spine: T8 (88\%), T9 (91\%), and T10 (85\%). It is interesting that DISH occurs at these sites. Hirasawa et al. and Kagotani et al. also found that DISH frequently occurred at T7-10 [12, 13]. Hirasawa et al. reported that more than $80 \%$ of DISH occurred at $\mathrm{T} 8 / 9$ or $\mathrm{T} 9 / 10$, which may reflect an anatomical effect; these vertebrae are susceptible to compressive mechanical stress because $\mathrm{T} 8$ is located almost at the peak of the physiological spinal kyphosis. Accordingly, DISH seems to arise mainly from the thoracic spine and may extend to the cervical and/or lumbar spine because of mechanical stress. Similar results were obtained in this study when the peak of spinal kyphosis was evaluated by supine CT. Interestingly, most of the ossification occurred to the right anterior of the vertebral body, and there were few ossifications in the area in contact with the arteries and veins. Previous study also demonstrated that the location of ossification was termed as anteolateral or right-sided hyperostosis [10]. Arterial blood flow and blood pressure may be affecting the ossification progress of DISH. Unfortunately we do not know about this mechanism from this study. However, it is for the first time report that we have studied the location of ossification in detail using the axial images of whole spine CT.

To date, the current knowledge on the pathogenesis of DISH is very limited. Some groups have reported that the pathogenesis of DISH is based on the excess of growth factors that might induce transformation of mesenchymal cells into fibroblasts and osteoblasts [20-22]. We think that it is need further analysis of these molecular mechanisms, which is important to clarify the etiology of DISH in the future. Okada et al. reported that the thoracolumbar junction (T11-L2) was the most frequently fractured level (54.8\%) in patients with DISH [1]. The fact that vertebral body fractures associated with DISH are more frequent in the thoracolumbar junction may be related to the fact that most of DISH occurs from T8 to T10. However, in this cross-sectional study, it was impossible to evaluate whether DISH occurred first in the thoracic spine and then secondarily in the cervical or lumbar spine.

This study has some limitations. First, we did not have access to clinical data such as the presence of diabetes, which is a risk factor associated with DISH. This was a retrospective study and it was not possible to investigate all subjects. Second, there may have been selection bias in the inclusion of subjects due to the fact that they were all trauma patients, but we did not have access to data about each patient before their trauma. DISH patients are usually asymptomatic, and health checks of the general population will be an excellent way to evaluate the prevalence of DISH. Finally, the radiation exposure experienced in whole-spine CT imaging in normal subjects is a health risk that must be considered. Some researchers agree that, when used correctly, such scans can save lives. However, according to some estimates, the radiation exposure a patient receives from a full-body CT scan is often 500 times that of an X-ray. Despite these limitations, our data suggest that the prevalence of DISH in Japan is larger than in past reports. We believe that prospective multicenter studies are needed to determine the prevalence and pathogenesis of DISH.

\section{Conclusions}

The prevalence of DISH based on whole-spine CT was $19.5 \%$, which is similar to that reported by other studies. Most ossification occurred at levels T8-10 and in the right anterior region of the vertebral body. 


\section{Additional file}

Additional file 1: Previous of DISH in each investigative modality. $\mathrm{N}$ : number, Xp: x-ray, AP: anterior-posterior, PA: posterior-anterior, CT: computed tomography. (DOC $47 \mathrm{~kb}$ )

\section{Abbreviations}

CT: Computed tomography; DISH: Diffuse idiopathic skeletal hyperostosis; ROAD: Research on Osteoarthritis/Osteoporosis Against Disability

\section{Funding}

Non financial associations that may be relevant or seen as relevant to the submitted manuscript.

Availability of data and materials

Data available upon request from corresponding author.

\section{Authors' contributions}

All authors have read and approved the manuscript. AH conceived and designed the study and interpreted the results; HK analyzed the data and interpreted the results; DS acquired the data and interpreted the results; MS acquired the data and interpreted the results; MT analyzed and acquired the data; MW conceived and designed the study and interpreted the results.

\section{Ethics approval and consent to participate}

This investigation was designed as a single-center retrospective comparative study and was approved by the Committee on Ethics and the Institutional Review Board of Tokai University School of Medicine (17R239). We have obtained informed consent form with opt-out method from patients.

\section{Competing interests}

The authors AH, HK, DS, MS, MT and MW declare that they have no competing interests.

\section{Publisher's Note}

Springer Nature remains neutral with regard to jurisdictional claims in published maps and institutional affiliations.

Received: 4 February 2018 Accepted: 22 May 2018

Published online: 30 May 2018

\section{References}

1. Okada E, Tsuji T, Shimizu K, Kato M, Fukuda K, Kaneko S, Ogawa J, Watanabe K, Ishii K, Nakamura M, et al. CT-based morphological analysis of spinal fractures in patients with diffuse idiopathic skeletal hyperostosis. J Orthop Sci. 2017:22(1):3-9.

2. Balling $H$, Weckbach $A$. Hyperextension injuries of the thoracolumbar spine in diffuse idiopathic skeletal hyperostosis. Spine. 2015:40(2):E61-7.

3. Rustagi T, Drazin D, Oner C, York J, Schroeder GD, Vaccaro AR, Oskouian RJ, Chapman JR. Fractures in spinal ankylosing disorders: a narrative review of disease and injury types, treatment techniques, and outcomes. J Orthop Trauma. 2017;31(Suppl 4):S57-74

4. Resnick D, Shaul SR, Robins JM. Diffuse idiopathic skeletal hyperostosis (DISH): Forestier's disease with extraspinal manifestations. Radiology. 1975; 115(3):513-24.

5. Littlejohn GO. Insulin and new bone formation in diffuse idiopathic skeletal hyperostosis. Clin Rheumatol. 1985:4(3):294-300.

6. Vezyroglou G, Mitropoulos A, Antoniadis C. A metabolic syndrome in diffuse idiopathic skeletal hyperostosis. A controlled study. J Rheumatol. 1996;23(4): 672-6.

7. Julkunen $\mathrm{H}$, Heinonen $\mathrm{OP}$, Knekt P, Maatela J. The epidemiology of hyperostosis of the spine together with its symptoms and related mortality in a general population. Scand J Rheumatol. 1975;4(1):23-7.

8. Kim SK, Choi BR, Kim CG, Chung SH, Choe JY, Joo KB, Bae SC, Yoo DH, Jun JB. The prevalence of diffuse idiopathic skeletal hyperostosis in Korea. J Rheumatol. 2004;31(10):2032-5.

9. Resnick D, Niwayama G. Radiographic and pathologic features of spinal involvement in diffuse idiopathic skeletal hyperostosis (DISH). Radiology. 1976;119(3):559-68.
10. Mata S, Chhem RK, Fortin PR, Joseph L, Esdaile JM. Comprehensive radiographic evaluation of diffuse idiopathic skeletal hyperostosis: development and interrater reliability of a scoring system. Semin Arthritis Rheum. 1998;28(2):88-96.

11. Cassim B, Mody GM, Rubin DL. The prevalence of diffuse idiopathic skeletal hyperostosis in African blacks. Br J Rheumatol. 1990;29(2):131-2.

12. Hirasawa A, Wakao N, Kamiya M, Takeuchi M, Kawanami K, Murotani K, Matsuo T, Deie M. The prevalence of diffuse idiopathic skeletal hyperostosis in Japan - the first report of measurement by $C T$ and review of the literature. J Orthop Sci. 2016;21(3):287-90

13. Kagotani R, Yoshida M, Muraki S, Oka H, Hashizume H, Yamada H, Enyo $Y$, Nagata K, Ishimoto $Y$, Teraguchi $M$, et al. Prevalence of diffuse idiopathic skeletal hyperostosis (DISH) of the whole spine and its association with lumbar spondylosis and knee osteoarthritis: the ROAD study. J Bone Miner Metab. 2015:33(2):221-9.

14. Weinfeld RM, Olson PN, Maki DD, Griffiths HJ. The prevalence of diffuse idiopathic skeletal hyperostosis (DISH) in two large American Midwest metropolitan hospital populations. Skelet Radiol. 1997;26(4):222-5.

15. Westerveld LA, van Ufford HM, Verlaan JJ, Oner FC. The prevalence of diffuse idiopathic skeletal hyperostosis in an outpatient population in the Netherlands. J Rheumatol. 2008;35(8):1635-8.

16. Mori K, Kasahara T, Mimura T, Nishizawa K, Nakamura A, Imai S. Prevalence of thoracic diffuse idiopathic skeletal hyperostosis (DISH) in Japanese: results of chest CT-based cross-sectional study. J Orthop Sci. 2017;22(1):38-42.

17. Oudkerk SF, de Jong PA, Attrach M, Luijkx T, Buckens CF, Mali WP, Oner FC, Resnick DL, Vliegenthart R, Verlaan JJ. Diagnosis of diffuse idiopathic skeletal hyperostosis with chest computed tomography: inter-observer agreement. Eur Radiol. 2017:27(1):188-94.

18. Laroche M, Moulinier L, Arlet J, Arrue P, Rousseau H, Cantagrel A, Mazieres B. Lumbar and cervical stenosis. Frequency of the association, role of the ankylosing hyperostosis. Clin Rheumatol. 1992;11(4):533-5.

19. Sarzi-Puttini P, Atzeni F. New developments in our understanding of DISH (diffuse idiopathic skeletal hyperostosis). Curr Opin Rheumatol. 2004;16(3): 287-92

20. Iwasawa T, Iwasaki K, Sawada T, Okada A, Ueyama K, Motomura S, Harata S, Inoue I, Toh S, Furukawa Kl. Pathophysiological role of endothelin in ectopic ossification of human spinal ligaments induced by mechanical stress. Calcif Tissue Int. 2006;79(6):422-30

21. Denko CW, Malemud CJ. Body mass index and blood glucose: correlations with serum insulin, growth hormone, and insulin-like growth factor-1 levels in patients with diffuse idiopathic skeletal hyperostosis (DISH). Rheumatol Int. 2006:26(4):292-7.

22. Eckertova M, Krskova K, Penesova A, Radikova Z, Zlnay D, Rovensky J, Zorad S. Impaired insulin secretion and uptake in patients with diffuse idiopathic skeletal hyperostosis. Endocr Regul. 2009;43(4):149-55.

\section{Ready to submit your research? Choose BMC and benefit from:}

- fast, convenient online submission

- thorough peer review by experienced researchers in your field

- rapid publication on acceptance

- support for research data, including large and complex data types

- gold Open Access which fosters wider collaboration and increased citations

- maximum visibility for your research: over $100 \mathrm{M}$ website views per year

\section{At BMC, research is always in progress.}

Learn more biomedcentral.com/submissions 